\title{
Experimental Validation of Multiple Attractors in a Simple Chaotic Circuit
}

\author{
FAWWAZ ABU KHADRA \\ Department of Electrical Engineering, Faculty of Engineering, University of Hafr Al Batin \\ Al Jamiah, Hafar Al Batin 39524, SAUDI ARABIA \\ Tel: (966) 137205488
}

\begin{abstract}
In this paper, a simple jerk circuit that allows studying the dynamical behavior of a three-dimensional autonomous chaotic system with only one nonlinear term is further investigated by numerical simulations and experimental validation. Depending on a single tuning parameter, the chaotic system is theoretically studied using standard techniques such as equilibrium analysis, bifurcation diagram, and Lyapunov exponents. Subsequently, the circuit that models the chaotic system is implemented to validate theoretical prediction experimentally. Despite the simple structure of the jerk circuit, experimental study of Fourier spectra has shown that the jerk circuit displays complex dynamics characterized by periodic limit cycles and aperiodic strange attractors. In addition, the jerk circuit has exhibited a wide tuning range and experimental results have shown good agreement with theoretical prediction except for few cases where numerical simulation has failed to accurately match experimental results due to sensitivity to initial conditions which is a signature of chaotic nonlinear systems.
\end{abstract}

Key-Words:- Chaotic systems, nonlinear systems, jerk circuits, bifurcation, Laypunov, Fourier spectra.

Received: February 3, 2020. Revised: July 25, 2020. Accepted: August 26, 2020. Published: August 30, 2020

\section{Introduction}

Since Lorenz proposed the first chaotic system in 1963 [1], there has been a considerable interest in developing and analyzing various mathematical systems that exhibit chaos [2]. In 1976, the work conducted by Rossler renewed the interest in threedimensional dissipative dynamical systems. These Rossler systems have a simpler algebraic structure compared with Lorenz system [3]. Later, many Lorenz-based or Lorenz-like chaotic systems have been found, such as Chen system, Lü system, Liu system, Cai system, Tigan system, and Sprott systems. $[3,4]$. In addition, there has been an increasing interest in designing experiments that will verify the predicted mathematical results [2]. The development of Chua's circuit in 1983 and its many variants, launched a quest for other circuits that chaotically oscillate [5] and there has been a debate over which one is the simplest example of chaos [6]. The simplest and most common examples of chaos in continuous-time systems come from three first-order (3D) ordinary differential equations with no explicit time dependence. Such equations are said to be autonomous [7]. Chaotic flows in autonomous 3D systems can be characterized as either dissipative or conservative, according to whether the trajectory is attracted to a region of space with fractal dimension less than 3, a so-called strange attractor [8]. Dissipative systems usually produce strange attractors independent of the initial conditions provided they lie in the basin of attraction [7, 8]. Two categories of autonomous 3D chaotic circuits include: (i) chaotic oscillators described by three first-order ordinary differential equations (ODEs), e.g. the wellknown Chua's circuit, and (ii) chaotic jerk oscillators described by a single third-order ODE called a jerk equation [9]. Chaotic systems written as jerk equations offer an exceptionally simple notation for higher order systems [6] as in Sprott systems [5,7,10]. The thirdorder ordinary differential equations of the jerk systems are written in the form $\dddot{x}=J(\ddot{x}, \dot{x}, x)$ whose solutions are chaotic [5]. The nonlinear function $J$ is called a "jerk," because it describes the third-time derivative of $x$, which would correspond to the time derivative of acceleration in a mechanical system $[5,8]$. A generalization of the simplest autonomous chaotic system is given by [10] as

$$
\dddot{x}+\ddot{x}+x+f(\dot{x})=0
$$

Where $f(\dot{x})$ is the nonlinear function required for chaos.

\section{Jerk Circuit Under Consideration}

Figure 1 shows the electric circuit under consideration which was proposed by Sprott [5]. The circuit consists of three successive active integrators in a feedback loop plus a second nonlinear feedback loop involving only two of the integrators and an inverter with a diode. To obtain the state equations of the jerk circuit, we use passive sign convention of RC circuit and kirchhoff's current law (KCL) as follows: After letting $\dot{x}=y$ and $\ddot{x}=z$, we get 


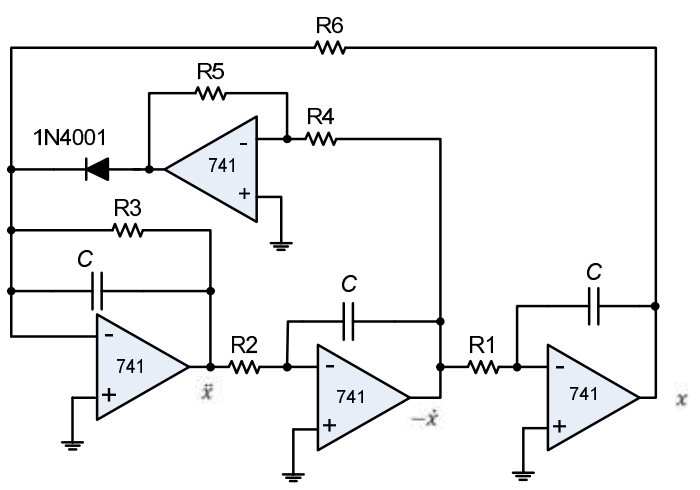

Figure 1. Chaotic Jerk Circuit under Consideration

$$
\left.\begin{array}{c}
\frac{d x}{d t}=\frac{y}{R_{1} C} \\
\frac{d y}{d t}=\frac{z}{R_{2} C} \\
\frac{d z}{d t}=-\frac{x}{R_{6} C}-\frac{I_{s}}{C}\left(\exp \left(\frac{y}{V_{T}}\right)-1\right)-\frac{z}{R_{3} C}
\end{array}\right\}
$$

The current $I_{S}$ is the junction scale or saturation current, its value is assumed to be $10^{-12} \mathrm{~A}$. The thermal voltage is typically assumed to have a value of $V_{T}=26 \mathrm{mV}$. The circuit was constructed in breadboard using standard components as indicated in schematic diagram of the circuit under investigation. The value of $R_{6}$ is variable since it used here as tuning parameter. The selection of $R_{3}=2 \mathrm{k} \Omega$ will produce maximum chaos when all other resistors in the circuit have a value of $1 \mathrm{k} \Omega$ as indicated by [5]. In this work, the tuning parameter is selected to be $\alpha=1 / R_{6}(\mathrm{k} \Omega)$ and $R_{3}$ is kept at $2 \mathrm{k} \Omega$. To further simplify the notation of the state equations, the measuring time is made in units of millisecond by selecting the value of all capacitors as $1 \mu \mathrm{F}$ and the value of all resistors in $\mathrm{k} \Omega$. In this case, it is necessary to multiply the nonlinear function $f(y)$ by a factor $R / R$. The state equation of the jerk circuit after this modification will become as

$$
\left.\begin{array}{c}
\dot{x}=y \\
\dot{y}=z \\
\dot{z}=-\alpha x-10^{-9}\left(\exp \left(\frac{y}{0.026}\right)-1\right)-0.5 z
\end{array}\right\}
$$

The existence of attractors in this model could be examined by estimating the volume contraction rate [11], which is described by the Lie derivative [12].

$$
\nabla V=\frac{\partial \dot{x}}{\partial x}+\frac{\partial \dot{y}}{\partial y}+\frac{\partial \dot{z}}{\partial z}=-0.5
$$

This negative value signifies that the system (3) is dissipative and there will be a fast exponential shrink of the volume in state space. Thus, the volume will infinitely converge to zero and the asymptotic motion through the flow will settle onto an attractor.

The equilibrium points of the system (3) can be obtained by finding its roots by setting $(\dot{x}=0, \dot{y}=$ $0, \dot{z}=0$ ). Obviously, it has only one equilibrium point at the origin $P_{0}(0,0,0)$. The dynamical behavior of the equilibrium point can be studied by computing the eigenvalues of the Jacobian matrix $J[11,13]$ of the system (3). The Jacobian matrix at the equilibrium point $P_{0}$ is expressed as:

$$
J(0,0,0)=\left[\begin{array}{ccc}
0 & 1 & 0 \\
0 & 0 & 1 \\
-\alpha & -\frac{10^{-9}}{0.026} & -0.5
\end{array}\right]
$$

The eigenvalues are determined by solving the characteristic equation, $\operatorname{det}[J-\lambda I]=0$, which is

$$
\lambda^{3}+0.5 \lambda^{2}-\frac{10^{-9}}{0.026} \lambda+\alpha=0
$$

Based on the Routh-Hurwitz stability criterion [3], which is not fulfilled in this case because eigenvalues have positive real roots, the equilibrium point $P_{0}$ is unstable and thus the system generates self-excited oscillations [11]. For example, when $\alpha=1$ where the system displays maximum chaos according to [5], the eigenvalues evaluated at $P_{0}$ are -1.197 and $0.3487 \pm$ j0.8447. Positive real roots of the eigenvalues guarantee instability. The dominant frequency of oscillation is expected to be at $f=844.7 / 2 \pi=$ $67.22 \mathrm{~Hz}$. This frequency is lower than the $75 \mathrm{~Hz}$ experimentally observed and almost the same value obtained using matlab simulation as shown in Figure 2. One main reason for the discrepancy between measured and simulated output is from tolerance values of the components used in the circuit. All measurements were captured and analyzed in the laboratory using Cassy Lab 2 data acquisition software. Notice that due to intense chaos, the frequency spectrum has broadband with significant power in harmonics. It was observed that the one-sided fast Fourier transform (FFT) obtained using Cassy Lab 2 has amplitude spectra higher than the simulated one by approximately $45 \%$ when the time step size is chosen to be $5 \mu \mathrm{sec}$. 


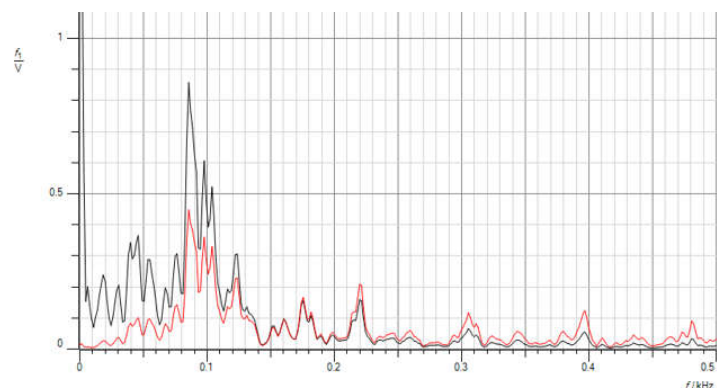

Figure 2(a). Experimental Frequency Spectrum of System (3) for $\alpha=1$.

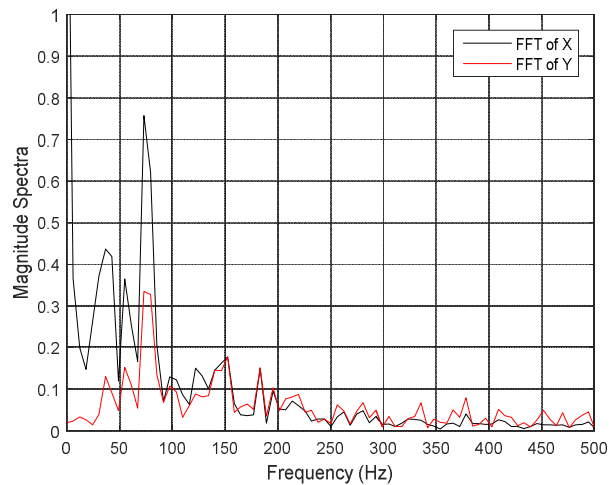

Figure 2(b). Simulation of Frequency Spectrum of System (3) for $\alpha=1$.

To investigate the influence of the tuning parameter $\alpha$ on the peak frequency produced, it is plotted against the peak frequency in Figure 3. The results indicate that as $\alpha$ increases (R6 decreases), the peak oscillation frequency increases but still in the very low frequency range. Of course, we can increase this frequency range by tuning the values of the capacitors.

Simulation of systems described by coupled first order differential equations, is usually carried out by the well-known fourth-order Runge-Kutta integrator implemented by ode45 function in matlab. The simulation was performed using absolute and relative error tolerances of $10^{-8}$. The simulation needs to be run for sufficiently long time (e.g., 100,000 points) and it is common practice to discard the transients related to initial conditions. The initial conditions are not critical just avoid the zero ones or you may select values close to the attractor once you have experimental output. It is not an easy task to match experimental and theoretical results because chaotic systems are extremely sensitive to initial conditions and during the experiment, we are not certain about the initial conditions since the starting voltage is provided by noise. For example, the time variation of the voltage $x$ and its derivative $y$ of the system (3) and the corresponding phase space plot of $y$ versus $x$ for $\alpha=$ 0.05 are shown in Figure 4 and Figure 5, respectively. The simulation was run for $500 \mathrm{msec}$ with step size of $5 \mu \mathrm{sec}$, and initial conditions were set close to zero ( 0 , $0.0001,0)$.

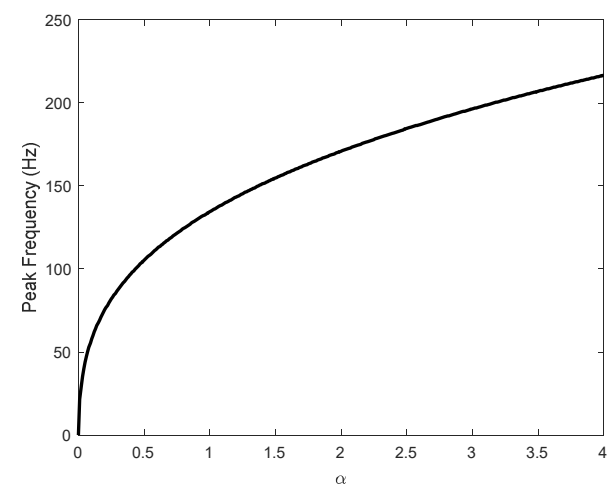

Figure 3. Peak Frequency against Tuning Parameter $\alpha$

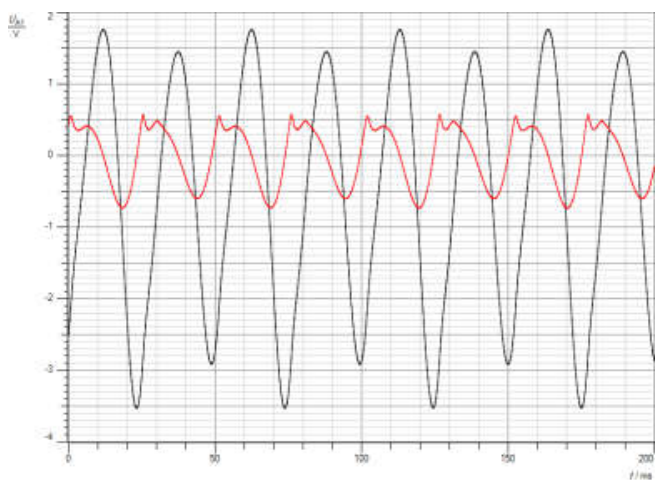

Figure 4(a). Experimental Time Variation of the Voltage $x$ and its Derivative $y$ of the System (3) for $\alpha$ $=0.05$.

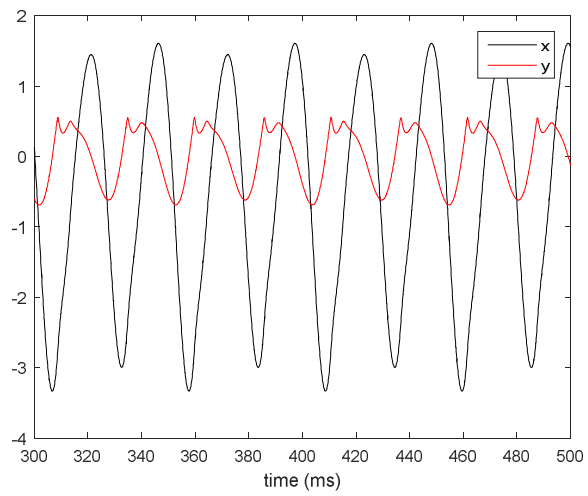

Figure 4(b). Simulation of Time Variation of the Voltage $x$ and its Derivative $y$ of the System (3) for $\alpha$ $=0.05$. 


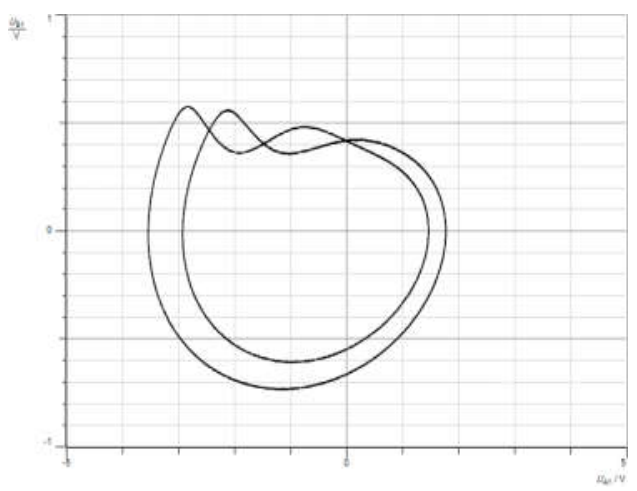

Figure 5(a). Experimental Phase Space of the Voltage $x$ and its Derivative $y$ of the System (3) for $\alpha=0.05$.

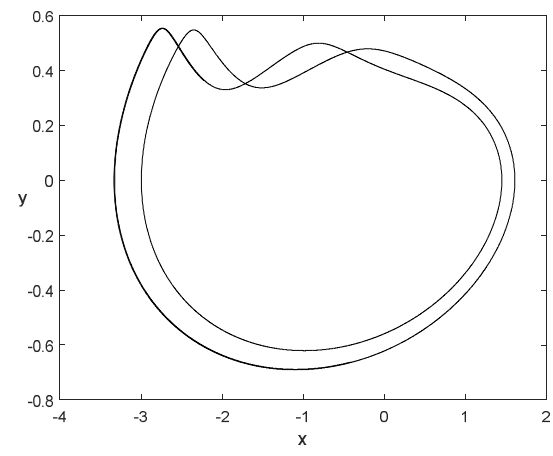

Figure 5(b). Simulation of Phase Space of the Voltage $x$ and its Derivative $y$ of the System (3) for $\alpha=0.05$.

\section{Route to Chaos}

The main characteristic of a chaotic systems is its sensitivity to initial conditions. A slight change in initial conditions result in very different results. There are several tools to study the dynamical behavior of chaotic systems such as Lyapunov exponent and bifurcation diagram. Evaluating the Lyapunov spectrum gives essential information to help distinguishing among various types of orbits that the chaotic system undergoes [14]. Based on Lyapunov spectrum of a third-order jerk system, there are four types of attractors.

- $\quad(\mathrm{LE} 1, \mathrm{LE} 2, \mathrm{LE} 3) \rightarrow(-,-,-)$ : fixed point.

- $\quad(\mathrm{LE} 1, \mathrm{LE} 2, \mathrm{LE} 3) \rightarrow(0,-,-)$ : limit point.

- $\quad(\mathrm{LE} 1, \mathrm{LE} 2, \mathrm{LE} 3) \rightarrow(0,0,-)$ : two-torus.

- $\quad($ LE1, LE2, LE3 $) \rightarrow(+, 0,-)$ : strange.

The bifurcation diagram displays the dynamics of a chaotic system and the route to chaos as a control parameter is varied in the system. Kaplan and Yorke defined the attractor dimension or Lyapunov dimension, as follows [15]:

$$
D_{k y}=j+\frac{\sum_{i=1}^{i=j} L E_{i}}{\left|L E_{j+1}\right|}
$$

where $j$ is the largest integer such that $\sum_{i=1}^{i=j} L E_{i} \geq 0$ and $\sum_{i=1}^{i=j+1} L E_{i}<0$.

To investigate the dynamics of the system (3), a preliminary simulation using Multisim was carried out to determine the range of the control parameter $\alpha$. It was found that the op amps in the circuit will get saturated for values of $R_{6}$ less than $200 \Omega$ this will set an upper limit of $\alpha$ to 5. To remain safe in the active region of the op amps, simulations and experiments were conducted to maximum value of 4 . Numerical study was performed by breaking down the range of $\alpha$ into three intervals to avoid long simulation and to be able to capture an approximate picture of the behavior of the chaotic system in (3). Figure 6(a) shows the bifurcation diagram of the system (3) of the value of $x$ when $\dot{x}$ is a local maximum versus the bifurcation parameter in the range $0 \leq \alpha \leq 0.25$. Figure 6(b) shows the bifurcation diagram of higher values of $\alpha$. In the range of $0.25 \leq \alpha \leq 2.5$, there was nothing special in the bifurcation diagram just pure chaotic. Finally, the higher range $2.5 \leq \alpha \leq 4$ is plotted in Figure 6(c).

The Lyapunov spectrum was calculated for the range $0 \leq \alpha \leq 4$ by breaking the range into eight intervals each with width of 0.5 then combined together as shown in Figure 7(a). Special attention has been paid for the low range as shown in Figure 7(b).

Based on the results shown in bifurcation diagram and Lyapunov spectrum, it is expected to have chaotic strange attractors for most of the values of $\alpha$ except for several points where the system exhibits limit cycles scenarios. To be concise, in the lower range of $\alpha$, a oneperiod limit cycle scenario for the range $0 \leq \alpha \leq 0.04$, period doubling at $0.04 \leq \alpha \leq 0.06$. Period-four is expected at $\alpha \cong 0.06$ and another limit cycle is expected in the range $0.2 \leq \alpha \leq 0.21$. Strange attractors are expected for the range $0.22 \leq \alpha \leq 2.86$. At higher range of $\alpha$, from Lyapunov spectrum and bifurcation diagram, we should have limit cycle for $2.86 \leq \alpha \leq 4$. The next step is to verify simulation results experimentally. This will be discussed next. 


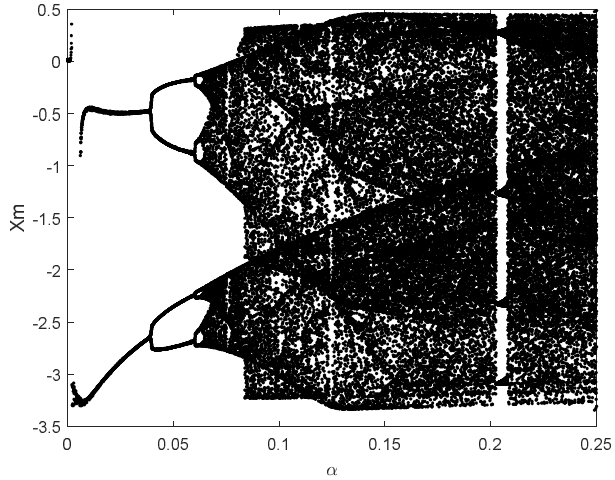

Figure 6(a). Bifurcation Diagram of the system (3) of the value of $x$ when $\dot{x}$ is a local maximum versus the bifurcation parameter $0 \leq \alpha \leq 0.25$.

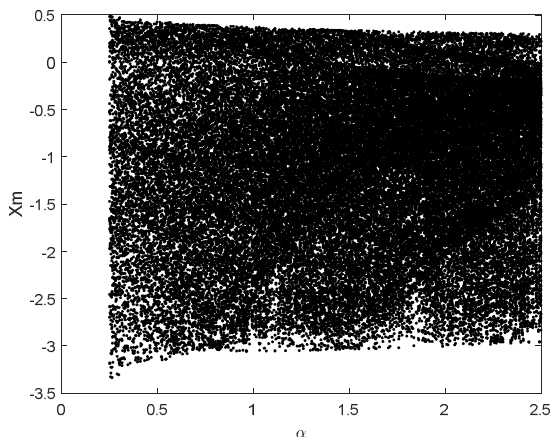

Figure 6(b). Bifurcation Diagram of the system (3) of the value of $x$ when $\dot{x}$ is a local maximum versus the bifurcation parameter $0.25 \leq \alpha \leq 2.5$.

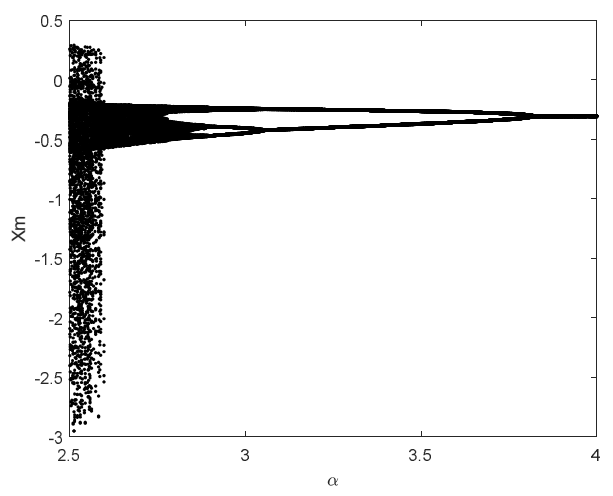

Figure 6(c). Bifurcation Diagram of the system (3) of the value of $x$ when $\dot{x}$ is a local maximum versus the bifurcation parameter $2.5 \leq \alpha \leq 4$.

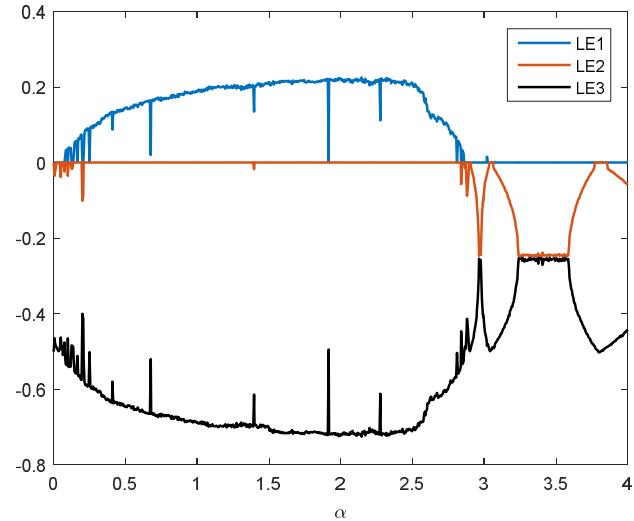

Figure 7(a). Lyapunov Spectrum of the System (3) for $0 \leq \alpha \leq 4$.

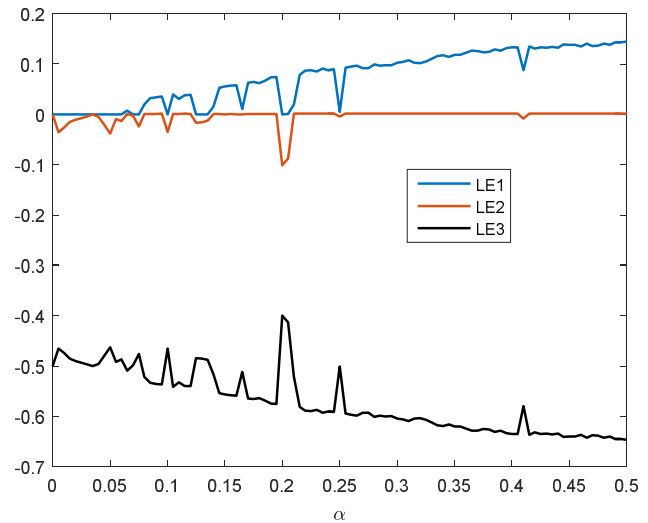

Figure 7(b). Lyapunov Spectrum of the System (3) for $0 \leq \alpha \leq 0.5$

\section{Experimental Results}

In order to verify the simulation results experimentally, the jerk circuit under study was constructed on breadboard using standard components. Resistors $R_{3}$ is fixed at $2 \mathrm{k} \Omega, R_{6}$ is tunable from 0.25 to $35 \mathrm{k} \Omega$, remaining resistors are with fixed value of $1 \mathrm{k} \Omega$, rectifier diode type 1N4001, and 741 op amps biased at $\pm 15 \mathrm{~V}$. Measurements were performed using Cassy Lab 2 data acquisition software. Measuring time was set to $500 \mathrm{msec}$ and the step size was set to $5 \mu \mathrm{sec}$. Experiment begins by setting $R_{6}=35 \mathrm{k} \Omega$ and gradually decreases its value while observing the output. The phase space trajectories, Fourier spectra, and time variation of $x$ and $y$ were captured at transition points. The time variation of $x$ and $y$ will be shown for selected values because they display somehow a similar pattern. The following scenarios were detected. 
For $25.8<R_{6}<35 \mathrm{k} \Omega$, a one-period limit cycle, twoperiod limit cycle in the range $13.6<R_{6}<25.8 \mathrm{k} \Omega$, four-period limit cycle in the range $12.7<R_{6}<13.6$ $\mathrm{k} \Omega$. Figure 8 shows the phase space and frequency spectrum for $R_{6}=35,25$, and $13.4 \mathrm{k} \Omega$, respectively. As the tuning resistor was further reduced, the following results were obtained: strange attractor in the range $4.9<R_{6}<12.6 \mathrm{k} \Omega$, two-period limit cycle in the range $4.5<R_{6}<4.9 \mathrm{k} \Omega$, and strange attractor in the range $1<R_{6}<4.5 \mathrm{k} \Omega$.

Figure 9 shows the phase space for $R_{6}=$ $5,4.9$, and $4.5 \mathrm{k} \Omega$, respectively. It can be concluded that a close agreement between theoretical and practical results has been achieved when the tuning parameter $\alpha$ is less than 0.5 .

Furthermore, from Lyapunov spectrum and bifurcation diagram, it is expected to experience strange attractors for $0.5<\alpha<2.85$ and limit cycles for $\alpha>2.85$. The results obtained are again very close to theoretical ones except for few points. The attractor begins to change its pattern for $\alpha>0.6$ and strange attractors of the second type were produced for up to $\alpha=3.45\left(R_{6}=\right.$ $290 \Omega)$ except at $\alpha=0.76\left(R_{6}=1.32 \mathrm{k} \Omega\right)$ and $\alpha=$ $1.0753\left(R_{6}=0.93 \mathrm{k} \Omega\right)$, a one-period limit cycle was experimentally produced against theoretical prediction. For $\alpha>3.45$, a one-period limit cycle of another different attractor has been produced down to the rest of the range while the op amps are still operating in the active region. The peak frequency of this attractor was surprisingly high $(500 \mathrm{~Hz})$ compared to other values and this frequency slightly increased as we decrease $R_{6}$. Figure 10 shows the phase space for $R_{6}=1.32,1$, and $0.93 \mathrm{k} \Omega$, respectively. Moreover, the time variation of $x$ and $y$ for $\alpha>3.45$ has no intersection and they have a sort of repulsive nature as shown in Figure 11. Finally, the op amps in the circuit went into saturation when reducing $R_{6}$ below $200 \Omega$. From the frequency spectrum produced, it is clear that limit cycles are of periodic nature and strange attractors have aperiodic spectrum. Furthermore, the frequency produced when $R_{6}$ has lower value than $290 \Omega$ was very high, reason could be due the op amps nonlinearity. Theoretical and experimental results for selected values are summarized in Table1.
Table 1. Summary of Theoretical and Experimental Results for Selected Values

\begin{tabular}{|c|c|c|c|c|}
\hline$\alpha$ & $\begin{array}{l}\text { Lyapunov } \\
\text { Exponents }\end{array}$ & Attractor & Dimension & $\begin{array}{c}\text { Peak } \\
\text { Frequency } \\
(\mathbf{H z})\end{array}$ \\
\hline 0.0286 & $\begin{array}{c}0,-0.0048,- \\
0.4956\end{array}$ & $\begin{array}{l}\text { One-period } \\
\text { Limit cycle }\end{array}$ & 1 & 32 \\
\hline 0.0400 & $\begin{array}{c}0,-0.0049,- \\
0.4954\end{array}$ & $\begin{array}{l}\text { Two-period } \\
\text { limit cycle }\end{array}$ & 1 & 37 \\
\hline 0.0746 & $\begin{array}{c}0.0177,0,- \\
0.5188\end{array}$ & $\begin{array}{c}\text { strange } \\
\text { (against } \\
\text { experimenta } \\
\text { 1) }\end{array}$ & 2.0341 & 48 \\
\hline 0.2000 & $\begin{array}{c}0,-0.1009,- \\
0.3996\end{array}$ & $\begin{array}{l}\text { Two-period } \\
\text { limit cycle }\end{array}$ & 1 & 60 \\
\hline 0.2041 & $\begin{array}{c}0.0053,- \\
0.0393,- \\
0.4660\end{array}$ & $\begin{array}{l}\text { Two-period } \\
\text { limit cycle }\end{array}$ & 1 & 62 \\
\hline 0.2222 & $\begin{array}{c}0.086,0,- \\
0.5871\end{array}$ & Strange & 2.1465 & 65 \\
\hline 0.7576 & $\begin{array}{c}0.1710, \\
0.0010,- \\
0.6719\end{array}$ & $\begin{array}{c}\text { Strange } \\
\text { (against } \\
\text { experimenta } \\
\text { 1) }\end{array}$ & 2.2545 & 80 \\
\hline 1.0000 & $\begin{array}{c}0.1895,0,- \\
0.6901\end{array}$ & Strange & 2.2746 & 87 \\
\hline 1.0753 & $\begin{array}{c}0.195, \\
0.0018,- \\
0.6966\end{array}$ & $\begin{array}{c}\text { Strange } \\
\text { (against } \\
\text { experimenta } \\
\text { 1) }\end{array}$ & 2.2799 & 80 \\
\hline 3.4483 & $\begin{array}{c}0,-0.2486,- \\
0.2521\end{array}$ & $\begin{array}{l}\text { One-period } \\
\text { limit cycle }\end{array}$ & 1 & 500 \\
\hline
\end{tabular}

\section{Conclusion}

In this paper, a relatively simple chaotic jerk circuit has been analyzed numerically and experimentally. Using Lyapunov exponents and bifurcation diagrams, dynamical properties of the chaotic systems under investigation has been categorized in terms of types of attractors produced as a result of tuning only one parameter. As a major contribution to this work, experimental results have shown that simple jerk circuits can exhibit unexpected behavior that differs from its theoretical complement. Furthermore, experimental verification of Fourier spectra of chaotic jerk circuits has contributed to understand the periodic and aperiodic nature of chaotic systems. Finally, randomness in chaotic jerk circuits can be utilized in the implementation of secure communication systems. This is to be considered as a potential future work. 


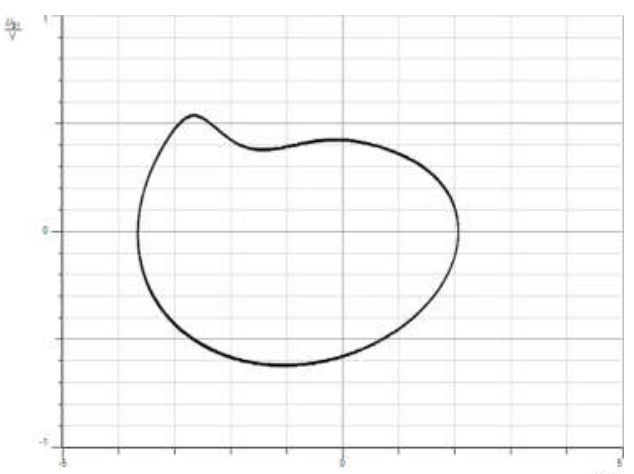

Figure 8(a). Phase space of $R_{6}=35 \mathrm{k} \Omega$.

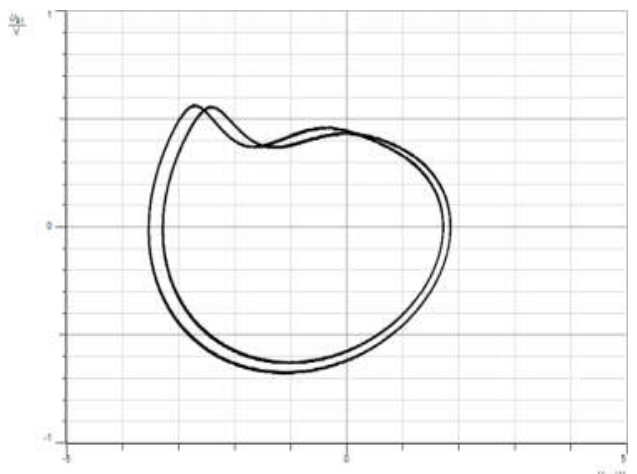

Figure 8(b). Phase space of $R_{6}=25 \mathrm{k} \Omega$.

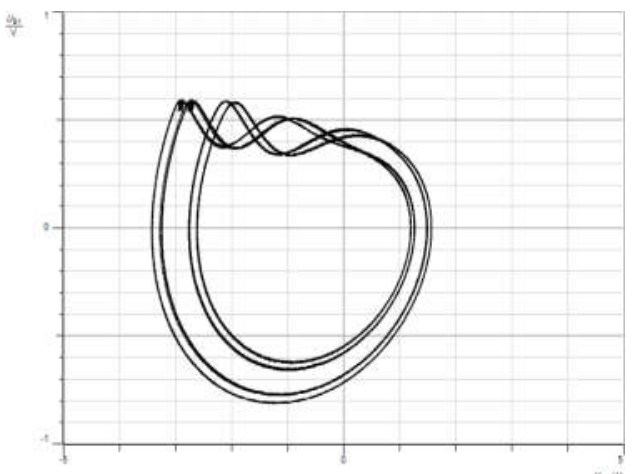

Figure 8(c). Phase space of $R_{6}=13.4 \mathrm{k} \Omega$.

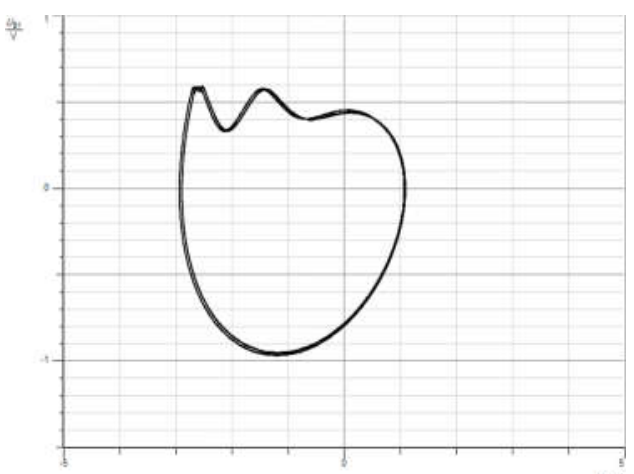

Figure 9(a). Phase space of $R_{6}=5 \mathrm{k} \Omega$.

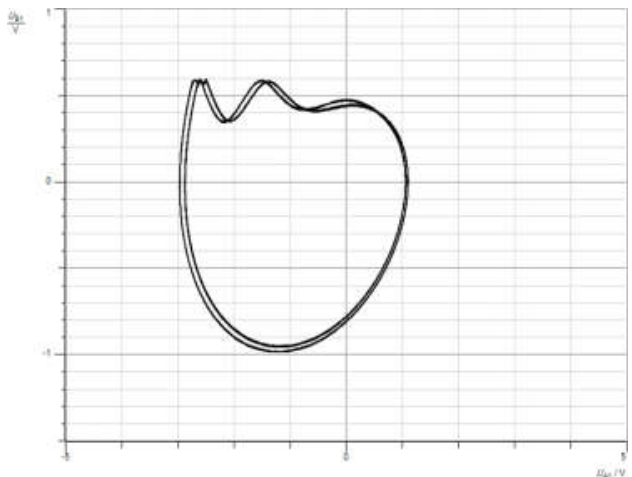

Figure 9(b). Phase space of $R_{6}=4.9 \mathrm{k} \Omega$.

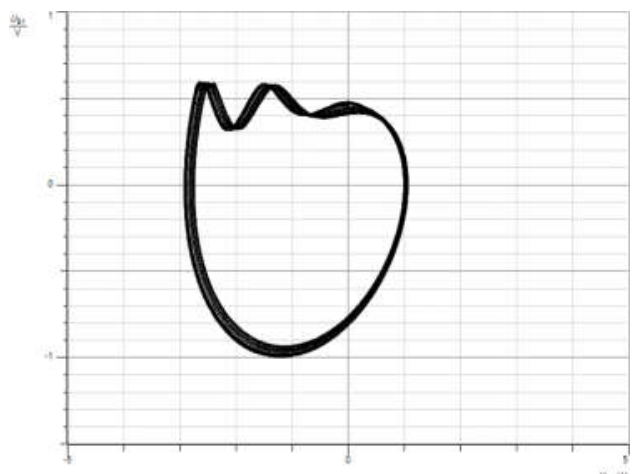

Figure 9(c). Phase space of $R_{6}=4.5 \mathrm{k} \Omega$. 


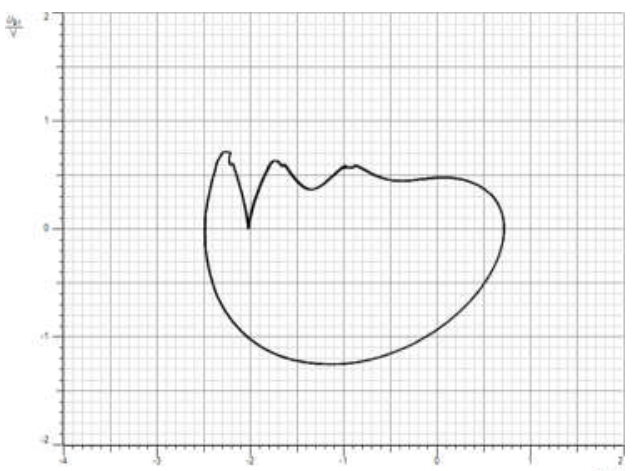

Figure 10(a). Phase space of $R_{6}=1.32 \mathrm{k} \Omega$.

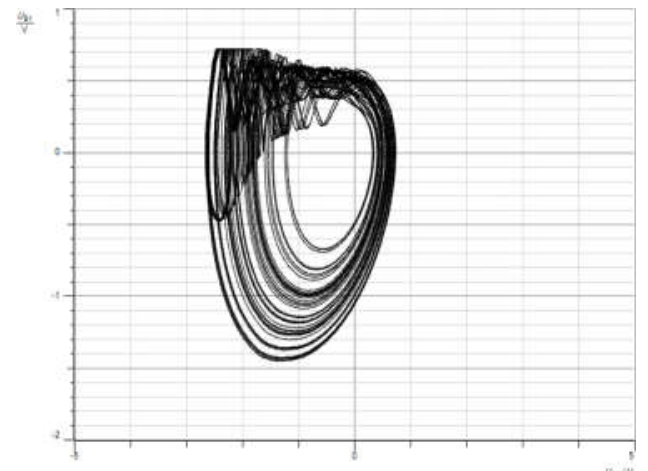

Figure 10(b). Phase space of $R_{6}=1.0 \mathrm{k} \Omega$.

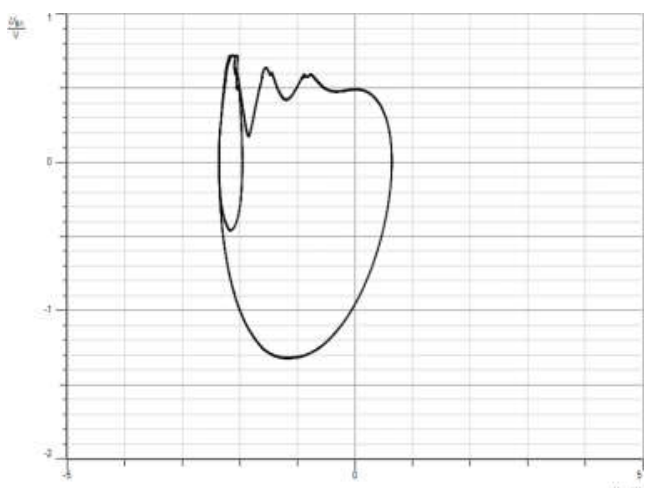

Figure 10(c). Phase space of $R_{6}=0.93 \mathrm{k} \Omega$.

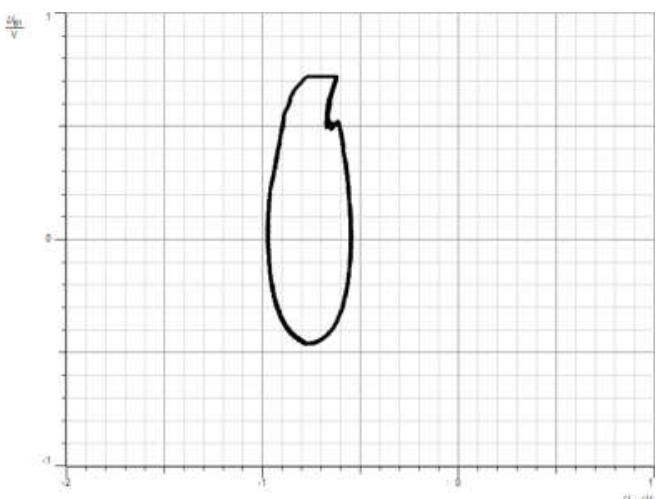

Figure 11(a). Phase space of $R_{6}=0.29 \mathrm{k} \Omega$.

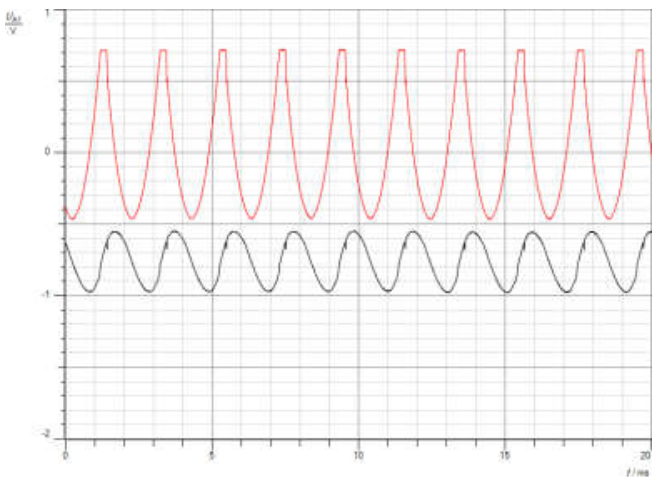

Figure 11(b). The Time Variation of the Voltage $x$ and its Derivative $y$ of $R_{6}=0.29 \mathrm{k} \Omega$.

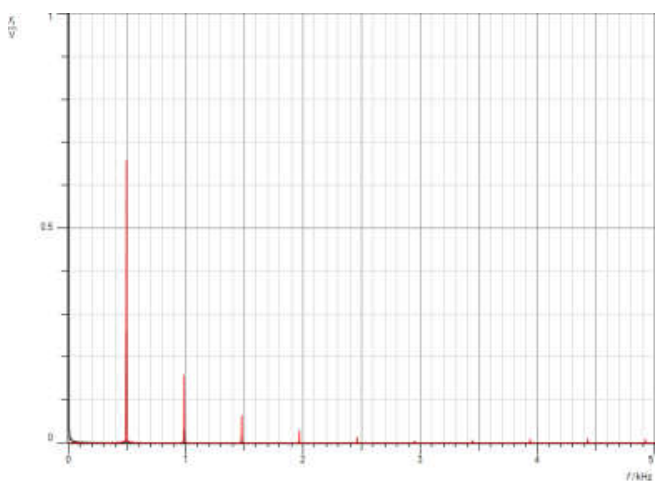

Figure 11(c). Frequency Spectrum of $R_{6}=0.29 \mathrm{k} \Omega$. 
References:

[1] Cui Yan, He Hongjun, Lu Chenhui, and Sun Guan. Finite Time Synchronization for Fractional Order Sprott C systems with Hidden Attractors. Complexity, https://doi.org/10.1155/2019/1612752.

[2] Er-Wei Bai, Karl E. Lonngren, J. C. Sprott. On the Synchronization of a Class of Electronic Circuits that Exhibit Chaos. Chaos Solitons \& Fractals, 13(2002), 1515-1521.

[3] Chunlai Li, Hongmin Li, Yaonan Tong. Analysis of a Novel Three-Dimensional Chaotic System. Optik 124 (2013), 1516-1522.

[4] Sundarapandian Vaidyanathan, A new 3-D jerk chaotic system with two cubic nonlinearities and its adaptive backstepping control. Archives of Control Sciences, 27(3), 2017, 409-439.

[5] J. C. Sprott. A New Chaotic Jerk Circuit. IEEE Transactions on Circuits and Systems-II: Express Briefs, vol. 58, No. 4, 240-243, April 2011.

[6] Jessica R. Piper and J. C. Sprott. Simple Autonomous Chaotic Circuits. IEEE transactions on circuits and systems-II: Express Briefs, vol. 57, No. 9, 730-734, September 2010.

[7] J. C. Sprott. Simplest Dissipative Chaotic Flow. Physics Letters A, 228 (1997), 271-274.

[8] J. C. Sprott. Some Simple Chaotic Jerk Functions. American Journal of Physics 65, 537 (1997), http://dx.doi.org/10.1119/1.18585.

[9] B. Srisuchinwong and D. Nopchinda. CurrentTunable Chaotic Jerk Oscillator. Electronics Letters 25th April 2013 Vol. 49, No. 9.

[10] Buncha Munmuangsaen, Banlue Srisuchinwong, and J.C. Sprott. Generalization of the Simplest Autonomous Chaotic System. Physics Letters A, 375 (2011), 1445-1450.

[11] G. H. Kom, J. Kengne, J. R. Mboupda Pone, G. Kenne, and A. B. Tiedeu. Asymmetric Double Strange Attractors in a Simple Autonomous Jerk Circuit.Complexity, https://doi.org/10.1155/2018/4658785.

[12] Wei Feng, Yi-Gang He, Chun-Lai Li, Xun-Min $\mathrm{Su}$, and Xiao-Qing Chen. Dynamical Behavior of a 3D Jerk System with a Generalized Memristive Device.Complexity, https://doi.org/10.1155/2018/5620956.

[13] Aceng Sambas, Mustafa Mamat, Zabidin Salleh, Mada Sanjaya WS, and Rizki Putra Prastio. Design, Numerical Simulation of Jerk Circuit and Its Circuit Implementation. Advanced Studies in theoretical Physics, Vol. 9, 2015, no. 7, 295-308, https://dx.doi.org/10.12988/astp.2015.410140.

[14] A. Sambas, M. Sanjaya W. S., and M. Mamat. Design and Numerical Simulation of Unidirectional Chaotic Synchronization and Its
Application in Secure Communication System. Recent Advances in Nonlinear Circuits: Theory and Applications. Journal of Engineering Science and Technology Review, 6(4) (2013), 66-73, ISSN: 1791-2377.

[15] A.E. Matouk and H.N. Agiza. Bifurcations, Chaos and Synchronization in ADVP Circuit with Parallel Resistor. J. Math. Anal. Appl. 341 (2008) 259-269.

\section{\&UHDMMH\&RP P RQVS WMEXWRQT/ IFHQMTDI

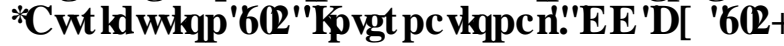

This article is published under the terms of the Creative Commons Attribution License 4.0 https://creativecommons.org/licenses/by/4.0/deed.en_US 